\title{
Closed-loop Architecture and Protocols for Rapid Dynamic Spreading Gain Adaptation in CDMA Networks
}

\author{
Lih-feng Tsaur, Daniel C. Lee \\ Department of Electrical Engineering, University of Southern California \\ Los Angeles, CA, 90089-2565 U. S. A. \{tsaur, dclee\}@usc.edu
}

\begin{abstract}
We present a closed-loop architecture and protocols for rapid dynamic spreading gain adaptation and fast feedback between a transmitter and a receiver communicating with each other in CDMA networks. These protocols and architecture do not require the transfer of an explicit control message indicating the change of CDMA spreading gain from transmitter to receiver. Also, with these protocols, the transmitter can change the spreading gain symbol-by-symbol as opposed to frame-by-frame, and feedback information (e.g., the fast-varying channel condition) can be exchanged almost as frequently as the symbol rate; thus, faster adaptation to the time-varying channel conditions of wireless networks and/or the rate variation of traffic is possible than with the existing frame-by-frame approach.
\end{abstract}

Keywords- CDMA, cellular networks, rate adaptation, information theory, system design

\section{INTRODUCTION}

Recently the multi-rate CDMA network has gained much attention for its use in multimedia applications, its immunity to multi-access interference (MAI), and its adaptability to channel conditions and data traffic variation [1-3]. One of the multi-rate communication networks $[2,4]$ that have been proposed uses the orthogonal/PN spreading scheme [5]. In this scheme, pseudonoise (PN) sequences [6] are used to create multiple frequencytime signal subspaces [7]. The interference from signals emanating from other frequency-time subspaces, which are spread by different PN sequences, is accordingly limited to the correlation between the PN sequences. Within the signal subspace, orthogonal sequences can be used to provide several orthogonal information links for users. In the current thirdgeneration cellular systems, orthogonal various spreading factor (OVSF) sequences [2] have been proposed for this purpose, as OVSF codes preserve the orthogonality between different spreading factors based on a modified Hadamard transformation [6].

In the past, to further improve the spectral efficiency and maintain the quality of service (QoS) of a CDMA system, several adaptive transmission schemes of adjusting signal waveforms were studied to support the system in time-varying wireless channels. These schemes include adjusting the transmitted power level [8][24], symbol rate [9], coding rate/scheme [10], constellation size [11], and any combination of these approaches [12-14]. Among the above schemes, a variation of adjusting the symbol rate was proposed recently, involving the application of dynamic spreading gain (SG) control
[15-17]. One advantage of SG control is that stations can adjust the spreading factor individually without affecting other links' communication quality of service, unlike some other schemes such as adjusting the transmission power.

In the OVSF/PN system, the symbol ${ }^{1}$ error probability can be adjusted by adjusting the OVSF code length while keeping the chip duration constant. We focus on such form of an SG control in the present paper. We can envision several ways to use such an SG adaptation (equivalently, symbol rate adaptation or symbol duration adaptation) in a CDMA network. For example, the system could have a centralized network controller making the decision about calls' temporal symbol transmission rates (spreading gains), or each call could decide its own temporal transmission rate for its rate adaptation. Regarding a scheme by which the transmitter and receiver agree on the spreading gain, we can envision a system in which the transmitter and receiver exchange explicit rate information or a system free of explicit messages containing the rate (spreading gain) information.

One simple SG control scheme would be to change the assignment of OVSF codes to different users dynamically according to channel or traffic condition. For example, a centralized controller at the base station could make such decisions, and we can imagine implementing code reassignment algorithms such as that proposed in [18] in such a way that the algorithms' execution could proceed extremely rapidly. Theoretically, this type of scheme can globally optimize the usage of OVSF code words for all stations in the system. However, this scheme would require a separate control channel to inform each station of the new OVSF code word, and such control messages would have be broadcast frequently, requiring substantial bandwidth. As a result, the spectral efficiency is greatly reduced.

Individual stations could adjust their data transmission rates separately according to individual stations' conditions. A transmitter can notify its peer receiver of such an adjustment through explicit rate information (RI) messages [2]. Since the latency of parsing these messages might be high, this scheme might not suit systems under fast time-varying conditions (e.g., fast time-varying fading channels).

In contrast to using RI messages, some studies [19] have proposed a message-free adaptation by using a blind rate-

\footnotetext{
${ }^{1}$ For readers not familiar with digital communication systems, word 'symbol' in this paper can be interpreted as 'bit'.
} 
detection algorithm for a slotted-time wireless network. In this design, the detector applies the Viterbi algorithm to detect the ending point of a data frame. The data rate is adjusted according to the utilization of the time slot. A disadvantage of this scheme is that bandwidth utilization is not maximized, since the time slots may not be fully occupied with data.

In [20], a symbol-by-symbol SG adaptation for a network operating in an OVSF/PN spreading CDMA system was studied for the first time. However, we found that lineages of the OVSF code tree presented in [21] posed difficulties in achieving blind rate detection. Namely, the receiver cannot distinguish between a symbol $S$ spread by a longer OVSF code sequence $[\mathrm{C},-\mathrm{C}]$ and two consecutive symbols $S,-S$ that are both spread by a shorter OVSF code $\mathrm{C}$. Here $[\mathrm{C},-\mathrm{C}]$ denotes the longer code sequence generated by concatenating $\mathrm{C}$ and $-\mathrm{C}$, and this code $[\mathrm{C},-\mathrm{C}]$ is a child of $\mathrm{C}$ in the binary OVSF code tree in [21]. For that reason, in [20] we had to devise a scheme to avoid generating such an $(S$, $-S$ ) symbol pair successively. However, this constraint may degrade performance. In order to remove such a constraint, in [22] we introduced a new structure of OVSF code sequences for fast symbol rate adaptation in the multi-rate CDMA system. We devised a new rule for generating such a codeword set and named it FOSSIL (Forest for OVSF-Sequence-Set-Inducing Lineages). Then we presented a protocol that allows fast spreading gain adaptation by using the OVSF sequences in FOSSIL. We note here that in order to execute this protocol, distributed entities (transmitter and receiver) need not send explicit control messages concerning the transmitter's change of spreading gain. The receiver detects the transmitter's behavior on the basis of data signals it receives.

In the present paper, we note that we may need a very fast feedback capability in order to fully utilize the benefits of the symbol-by-symbol spreading gain adaptation. For example, if we are to use the rapid adaptation capability to adapt to the fastvarying channel condition, the channel condition must be rapidly fed back to the transmitter. Our preliminary study [23] shows that by employing symbol-by-symbol spreading gain adaptation with perfect channel state information, the data throughput can be improved by orders of magnitude for a given symbol error rate requirement. The protocol presented in [22] does not illustrate the capability of our OVSF-based symbol-by-symbol spreading gain control approach to accommodate fast (symbolby-symbol) feedback. The present paper sets forth an extended system architecture and the protocols that allow both symbol-bysymbol spreading gain adaptation and fast feedback.

In section II, the core ideas of OVSF-based spreading gain adaptation and a receiver's spreading gain detection are illustrated. Section III discusses how FOSSIL can facilitate the allocation of code sets to multiple users in the CDMA network, with the goal of preserving orthogonality among user signals. Section III also introduces what we call a 'conjugate FOSSIL' structure, to be used for feedback capability. Section IV presents the protocols and system architecture extended for fast feedback.

\section{CORE FEATURES OF OVSF-BASED RATE-ADAPTIVE SYSTEM WITHOUT EXPLICIT RATE INFORMATION}

In this section, we present the core ideas of our OVSF-based fast spreading gain control that does not require the transmitter to send explicit messages indicating the current spreading gain. Throughout this paper, any spreading code sequence has a length that is a power of 2 , and each term in the code sequence takes value either 1 or -1 . We first define the "shift-orthogonality" of codes.

Definition: Code sequence $U$ of length $n$ and code sequence $V$ of length $n L$ are "shift orthogonal with unit length $n$ " iff $\sum_{k=1}^{n} U(k) V(n l+k)=0$ for each of $l=0,1, \cdots, L-1$. To assist our presentation, we denote each symbol by a complex number $A$. (Readers who are not familiar with the digital communication system can consider only binary symbols and view $A$ as a variable whose value can be either 1 or -1 .) The signal carrying a symbol $A$ before spreading at the transmitter side is represented during the symbol duration by a constant function of time $t$ :

$$
A, \tilde{t}<t<\tilde{t}+T
$$

where $\tilde{t}$ is the beginning of the symbol interval and $T$ is the symbol duration. The signal's waveform spread by a spreading code (binary sequence) $\mathrm{C}$ is represented by function $s(t)$ $=A w_{C}(t-\tilde{t})$, where function $w_{C}$ associated with spreading code $\mathrm{C}$ is

$$
\begin{aligned}
& w_{C}(t)=C[k], \quad(k-1) \Delta<t<k \Delta, \\
& \text { for } k=1,2, \ldots,|C|
\end{aligned}
$$

In this paper we denote by $|\mathrm{C}|$ the length of the spreading code $\mathrm{C}$, and by $\Delta$ the CDMA chip duration.

For simple illustration we start our discussion from the single-user rate-adaptive system.

\section{A. The Single-User Multirate Adaptive System}

In this system, the transmitter and receiver have a pre-agreed set of binary OVSF spreading codes with different lengths, $\mathcal{S}=$ $\left\{C_{1}, C_{2}, \ldots, C_{L}\right\}$; that is, any pair of codes, $C_{i}$ and $C_{j}$ are shiftorthogonal with unit length $\min \left(C_{i}, C_{j}\right)$. (For binary-tree constructed OVSF code trees, the lengths of the OVSF code sequences are all different powers of 2. An example of such code set can be found in the set of codes along the first-born lineage of FOSSIL to be presented in section III.) We refer to this shift-orthogonality property within a code set as IOVSF property (internal OVSF). We index these codes in a way that $\left|C_{j+1}\right|=2\left|C_{j}\right|$. When the transmitter wants to send a data symbol with duration $\left|C_{i}\right|$ chips, the transmitter spreads the data symbol with code $C_{i}$. (The spreading process is equivalent to modulating the data symbol with the code sequence's signal waveform [25].) The receiver can then sequentially parse the symbol intervals from the incoming signal by concurrently correlating the received signal with the codes in $\mathcal{S}$. (The correlating process is equivalent to demodulating the received signal's waveform [25].) By taking the advantage of the OVSF property within the codes set $\mathcal{S}$, the receiver determines the current received symbol's spreading code employed by the transmitter and thus determines the current symbol's duration. From the receiver's point of view, regarding symbol's spreading code, there are $L$ hypotheses; namely, $C_{1}, C_{2}, \ldots, C_{L}$. The receiver detects which of these codes have been used by the transmitter for each symbol on the basis of the correlation output.

We explain the receiver's detection process by simple illustration. For simplicity of receiver's detection process and for 
seeing only the essential feature of the scheme, we impose an additional rule to the transmitter. The transmitter maintains a modulo-counter $Y$. Starting from zero, $Y$ increases by one whenever one chip's duration of the data symbols elapses. $Y$ is reset to zero whenever its value reaches the length of the longest spreading codes in $\mathcal{S}$. The spreading code is used according to the following rule.

\section{Rule 1: (modulo counter rule)}

A symbol signal spread by Codeword $C_{i}$ can start only when ( $Y$ $\left.\bmod \left|C_{i}\right|\right)=0$.

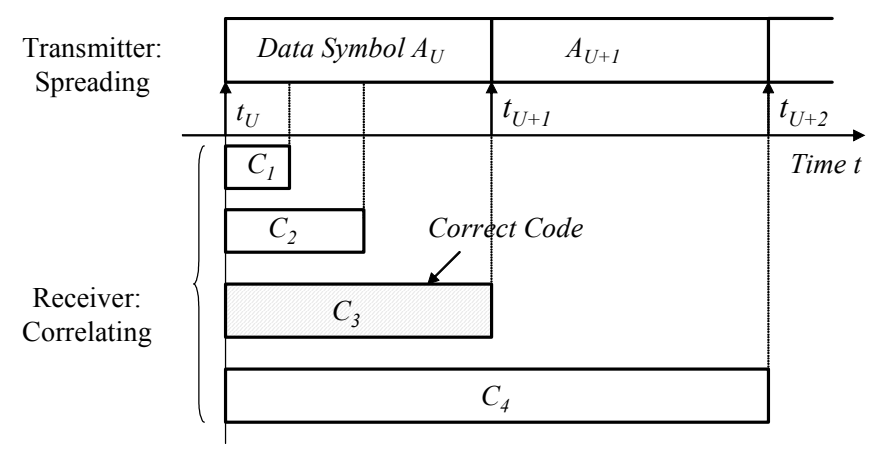

Figure 1. Receiver's decoding process

Fig. 1 illustrates the receiver's process of detecting the duration of a data symbol (denoted by $A_{U}$ ) under the assumption that the previous symbols' durations have been correctly detected. ${ }^{2}$ In this illustration, the code set used for the fast spreading gain control is $\left\{C_{1}, C_{2}, C_{3}, C_{4}\right\}$. The spread data symbol $A_{U}$ starts at $t_{U}$ at the receiver's side, and the next symbol $A_{U+1}$ starts at $t_{U+1}$. (The duration of symbol $A_{U}$ is $t_{U+1}-t_{U}=$ $\left|C_{3}\right| \Delta$.) Both symbols $A_{U}$ and $A_{U+1}$ are spread by the spreading code $C_{3}$. The received signal's waveform, denoted by $r(t)$ can be represented by

$r(t)=\left\{\begin{array}{r}A_{U} C_{3}[k]+n(t), \quad t_{U}+(k-1) \Delta<t<t_{U}+k \Delta, \\ \text { for } k=1,2, \ldots,\left|C_{3}\right| \\ A_{U+1} C_{3}[k]+n(t), t_{U+1}+(k-1) \Delta<t<t_{U}+k \Delta, \\ \text { for } k=1,2, \ldots,\left|C_{3}\right|\end{array}\right.$

where $n(t)$ represents noise.

At $t_{U}$ the receiver starts correlating the received signal with all codes $C_{1}, C_{2}, C_{3}$, and $C_{4}$; that is, the receiver performs operations:

$$
\frac{1}{\left|C_{i}\right| \Delta} \int_{t_{U}}^{t_{U}+\left|C_{i}\right| \Delta} r(t) w_{c_{i}}\left(t-t_{U}\right) d t
$$

for $i=1,2,3,4$. Due to the shift-orthogonality (OVSF) property of codes $C_{1}, C_{2}, C_{3}, C_{4}$ (and due to the modulo counter rule) only the correlation for case $i=3$ has positive value and the correlation is zero for $i=1,2,4$ in the absence of noise or distortion of the

\footnotetext{
${ }^{2}$ In a noisy environment, there is a positive probability that a receiver can make a detection error. In that case, the error can propagate to the detection of subsequent symbol durations. Such anomaly can be detected by other modules, and can be cured by symbol resynchronization.
}

received signal. As illustrated by this example, the spreading code used by the transmitter can be detected by the receiver without the explicit message sent from the transmitter. The detection will be error-free in the absence of noise and distortion. In the reality of noise or distortion, detection rules based on the correlations are left as design parameter of the system, which should depend on the communication environments. An example of a detection rule would be the maximum likelihood detection, which chooses code $C_{i}$ that yields the maximum correlation properly scaled. If we do not impose the modulo counter rule (Rule 1), the simple detection rule illustrated above fails. Fig. 2 illustrates how such failure occurs. However, we can design more complicated detection rules, which are again based on correlation with OVSF codes, that are valid even for a system without the modulo counter rule. In the present paper, we will limit our discussion to the system that enforces the modulo counter rule.

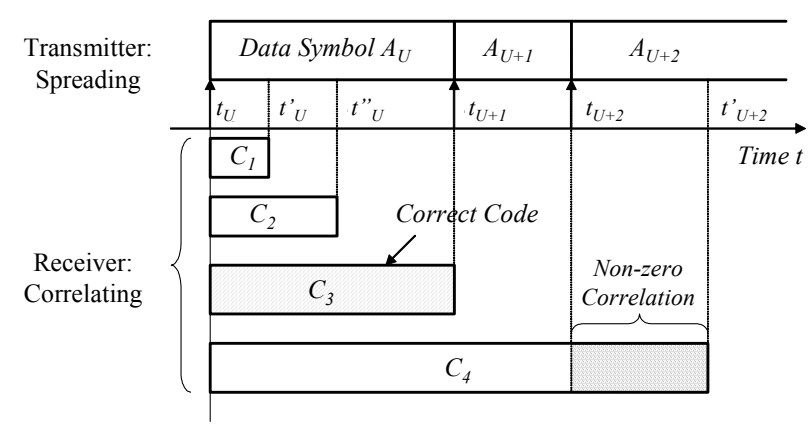

Figure 2. Without modulo counter rule

Finally, we note that in order to have spectral efficiency, multiple 'Single-User Multirate Adaptive Systems' can share the same frequency band by separating different user signals by different PN codes (scrambler). In such a OVSF/PN CDMA system, a user's symbols spread by the OVSF codes are again modulated by a PN code, as illustrated by Fig. 3 .

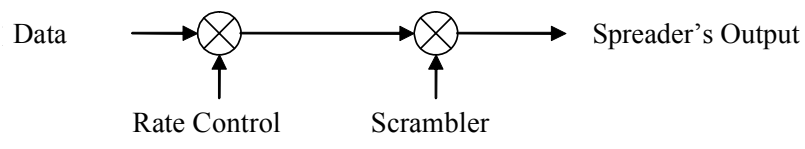

Figure 3. OVSF/PN system

\section{B. Point-to-Multipoint Multiuser Multirate Adaptive System}

We now progress further to present the multi-user multi-rate adaptive system, where multiple data sources simultaneously adjust their spreading gains in a shared communication channel without generating the mutual interference. An application of this system would be the multiple forward links of a CDMA-based cellular network. For this reason we will refer to the point-tomultipoint links used by different users as forward links. In fact, the forward links of the $3^{\text {rd }}$ generation cellular networks are channelized by the OVSF codes. The system described in this section adds the rapid (symbol-by-symbol in contrast to frameby-frame) spreading gain adaptation capability.

This system architecture is based on the design for the singlerate-adaptive system in 3.1. Every data source-sink pair is assigned with a pre-agreed spreading code-set. Similarly, the spreading code sets $\mathcal{S}_{i}$ for the $i$ th forward link contains the OVSF codes of different lengths. In addition, in order to separate 
signals in different forward links (to reduce mutual interference among forward links), we need the following rule:

Rule 2 (Code set allocation rule) : Every code in $\mathcal{S}_{i}$ and every code in $\mathcal{S}_{j}$ (code set for another forward link, $j$ ) must be shift-orthogonal to each other with unit length being the shorter of the two (i.e., OVSF).

As in the case of the $3^{\text {rd }}$ generation cellular networks, synchronization among the symbols transmitted through different forward links must be maintained in order to reduce interference among those links. Fig. 4 provides an example of the circumstances, where the ensemble signal's waveforms received by data sink 2 is decoupled into two individual waveforms that are respectively generated by the data source 1 and 2 .

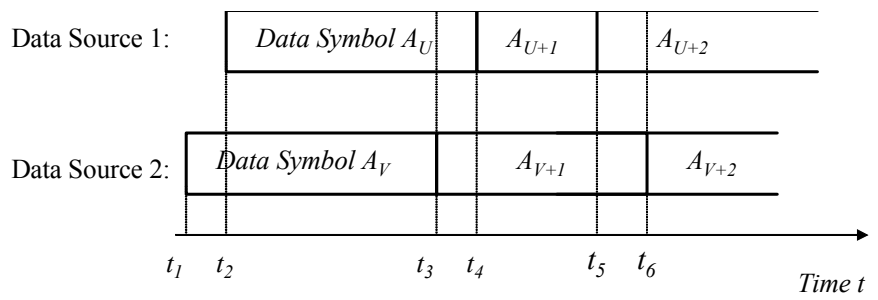

Figure 4. Loss of synchronism among links

In this example, when data sink 2 correlates the ensemble signal's waveform received between time $t_{3}$ and $t_{6}$, it will obtain the correlating output that contain the additional component leaked from the data source 1's signal waveform.

To maintain synchronization among different forward links, the modulo-counter $Y$ introduced in section II can be shared by all forward links, and can be maintained in the same manner as for the single rate-adaptive system. At all data sinks, the same modulo counter $Y$ is also implemented. In this case the modulo number should be the length of the longest spreading code used in all the forward links. Data source of forward link $i$ can only use codeword $C_{k}$ in $\mathcal{S}_{i}$ when $\left(Y \bmod \left|C_{k}\right|\right)=0$. Data sink of forward link $i$ correlates the received signal with the pre-agree codes in $\mathcal{S}_{i}$, and select the spreading code yielding the maximal correlation output as the current symbol's spreading code.

Finally, we need to discuss how we design codes sets and allocate them to different forwards links so that Rules 1, 2 above are satisfied. (In addition to these rules, the range of code lengths in a code set must be chosen commensurately to the range of symbol rates requested by the links.) In order to facilitate the generation and allocation of codes sets, we present our forest-structured code generation method in section III.

\section{The FOSSIL-BASED SPREADING CODE AND CODE SETS}

By spreading the data symbols respectively with the shiftorthogonal code sequences of different lengths, the central station can simultaneously transmit the data to different receivers without causing the mutual interferences. This concept has been adopted in the various WCDMA cellular system standards to create the multi-user, multi-rate communication in the forward channel. One generation scheme has been proposed in $[2,21]$. This tree-structured code generation has the following two important properties.
Property T1: Two codes, $C_{i}$ and $C_{j}$ in the code tree are shift orthogonal to each other with the unit length $\min \left(\left|C_{i}\right|,\left|C_{j}\right|\right)$ as long as one is not a descendant of the other.

Property T2: Two codes are not shift orthogonal if one is a descendant of the other.

Due to Property T2, if a channel is using a particular code word to spread data, other channels must avoid using any of this code word's descendants or ancestors to avoid interference between channels. For the purpose of symbol rate adaptation, nodes in the code tree can be grouped into several mutually exclusive branches, and the distinct branches can be assigned to different channels. Since a branch has code words with different lengths, a transmitter assigned with a branch can spread data with variable spreading factor, and thus enable multi-symbol-rate communication. Indeed, this code tree can be used for code set allocation for the $3^{\text {rd }}$ generation cellular networks enabling the 'frame-by-frame' symbol rate adaptation with 'explicit rate information' send for each frame from the transmitter to the receiver.

However, for our 'symbol-by-symbol' duration adaptation 'without explicit rate information' which was presented in section II, codes of different lengths used by the same link must be shift orthogonal too. Allocating a branch in code tree of [21] to a link would not enable the rapid rate adaptation presented in section II because codes of different lengths within a branch are not shift-orthogonal. Therefore, we present another code generation method, which has a 'forest' structure instead of 'tree' and named 'FOSSIL (Forest for OVSF-Sequence-Set Inducing Lineages). In section III.A, we will present the code generation through FOSSIL. In section III.B we note that FOSSIL facilitates the allocation of code sets that have the properties required for the rapid spreading gain (duration) adaptation in section II. In section III.C we present another code forest named conjugate FOSSIL which is associated with FOSSIL. Both FOSSIL and conjugate FOSSIL will be heavily used to add rapid feedback capability to the rapid spreading gain adaptation.

\section{A. Construction of FOSSIL}

FOSSIL was first introduced in [22], and interesting properties of FOSSIL were also presented and proved in [22]. A FOSSIL is generated from a number of root codes, $R_{1} \equiv[A$, $\mathrm{a}], \mathrm{R}_{2} \equiv[\mathrm{B}, \mathrm{b}], \mathrm{R}_{3} \equiv[\mathrm{C}, \mathrm{c}], \ldots$, where code word $[\mathrm{X}, \mathrm{X}]$ denotes concatenation of $\mathrm{X}$ and $\mathrm{x}$, both with half of the length; i.e., $|\mathrm{X}|=$ $|x|=1 / 2 \mid[X, x]$. All the halves (e.g., A, a, B, b, C, c, ...) must be distinct sequences with equal length that is a power of 2 . These root codes have the property that the first halves are orthogonal to one another, and so are the second halves; i.e. $A * B=A * C=$ $\mathrm{B} * \mathrm{C}=\ldots=0$, and $\mathrm{a} * \mathrm{~b}=\mathrm{a} * \mathrm{c}=\mathrm{b} * \mathrm{c}=\ldots=0$. (We denote by symbol $*$ the binary correlation of two sequences with the same length.) From these roots, FOSSIL is constructed by each node begetting two children. The rule is that node (representing code or sequence) $[\mathrm{U}, \mathrm{V}]$ begets $[\mathrm{U}, \mathrm{V}, \mathrm{U}, \mathrm{V}]$ and $[\mathrm{U},-\mathrm{V},-\mathrm{U}, \mathrm{V}]$, where $\mathrm{U}$ and $\mathrm{V}$ denote the first and the second halves of code $[\mathrm{U}, \mathrm{V}]$. We refer to [U, $-\mathrm{V},-\mathrm{U}, \mathrm{V}]$ as the 'first-born' of [U, V]. Thus, every code sequence in a FOSSIL has a length that is a power of 2. We define FOSSIL's 'first-born lineage' as the set of code words with distinctive lengths such that the code of length $2 n$ is the first-born child of the code of length $n$.

In our presentation of code trees, we denote by $C_{n}{ }^{i}$ the $i^{\text {th }}$ code of length $n$ in the code forest. In our illustration of FOSSIL in 
figures, index $i$ is a positive integer between 1 to $n$. To simplify the notation, we also refer a code sequence as a "node" in the code forest. For instance, we will sometimes use "node $C_{n}{ }^{i}$ ". The length of each code in FOSSIL is a power of 2 , so we can decompose $C_{n}{ }^{i}$ into two sequences of length $n / 2$. We denote by ${ }_{1} C_{n}{ }^{i}$ and ${ }_{2} C_{n}{ }^{i}$ the first and the second half of $C_{n}{ }^{i}$, i.e. $C_{n}{ }^{i}=\left[{ }_{1} C_{n}{ }^{i}\right.$, $\left.{ }_{2} C_{n}{ }^{i}\right]$ and $\left|{ }_{1} C_{n}{ }^{i}\right|={ }_{2} C_{n}{ }^{i}|=1 / 2| C_{n}{ }^{i} \mid$. In addition, we also denote by $F T_{1}, \ldots$, and $F T_{k}$ the FOSSIL code trees generated from root codes $R_{1}=$ [A, a], ..., and $R_{k}$, respectively, as shown in Fig. 5, where root code $R_{k}$ has length of $r$.
$F T_{n}$

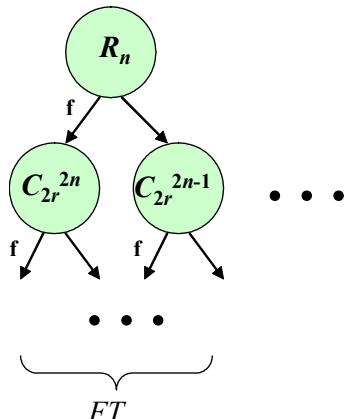

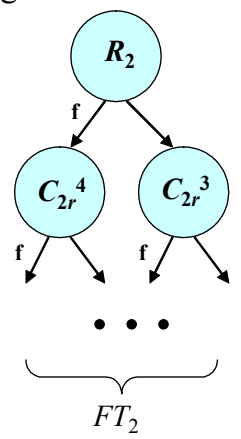

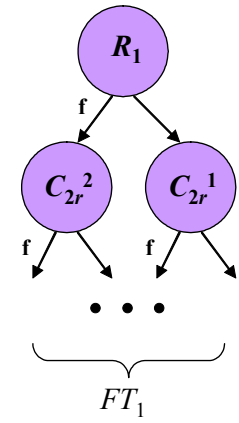

Direction in which code index is increasing
FOSSIL shares property $\mathrm{T} 1$ with the binary code tree in [21]. However, FOSSIL has the following convenient property:

Property F2: Two codes $C_{i}$ and $C_{j}$ in the same first-born lineage are shift orthogonal to each other with unit length $\min \left(\left|C_{i}\right|,\left|C_{j}\right|\right)$.

Also, the following properties of FOSSIL have been proven in [22].

Property F3: (Half-wise orthogonality): All FOSSIL codes of the same length are half-wise orthogonal to one another; that is, for each $n$ and any pair $i, j,{ }_{1} C_{n}{ }^{i}$, and ${ }_{1} C_{n}^{j}$ are orthogonal, and ${ }_{2} C_{n}{ }^{i}$ and ${ }_{2} C_{n}^{j}$ are orthogonal.

Property F4: A FOSSIL code of length $n$ is shift orthogonal with unit length $n$ to its first-born child and to any of the firstborn child's descendants.

\section{B. Allocation of Code-sets in FOSSIL}

In this section, we discuss the allocation of codes in FOSSIL to different users. By 'branch', we refer to a collection of a node (the patriarch) and all its descendants up to the $n$th generation. (Conceptually, we can allow $n=\infty$, allowing a branch with infinitely many nodes.) We note the following property of FOSSIL:

Condition for OVSF branches: Codes, $C_{i}$ and $C_{j}$, from different non-overlapping branches in FOSSIL are shiftorthogonal to each other with unit length $\min \left(\left|C_{i}\right|,\left|C_{j}\right|\right)$, as long as their patriarchs are not in ancestor-descendant relation or as long as one patriarch is a descendant of the other in its firstborn lineage.

Therefore, we can allocate such non-overlapping branches to different forward links without mutual signal interference. In fact, for the purpose of spreading gain adaptation in section II, the links only need to use the first-born lineage of each branch.

\section{Conjugate FOSSIL}

For the FOSSIL generated from the root codes $R_{1}=[\mathrm{A}, \mathrm{a}], R_{2}$ $=[\mathrm{B}, \mathrm{b}], R_{3}=[\mathrm{C}, \mathrm{c}]$, etc, the sequences $\pm[\mathrm{A},-\mathrm{a}], \pm[\mathrm{B},-\mathrm{b}], \pm[\mathrm{C}$, $-c]$, etc. do not appear in the resulting FOSSIL. In fact, they can be used as the root codes to generate different sets of codes with FOSSIL structure. In this paper, we are particularly interested in one alternative that is constructed from the root codes $[\mathrm{A},-\mathrm{a}],[\mathrm{B},-\mathrm{b}], \ldots$ etc. We call the resulting FOSSIL as the "conjugate FOSSIL" of the FOSSIL generated by $R_{1} \equiv[\mathrm{A}$, $\mathrm{a}], R_{2} \equiv[\mathrm{B}, \mathrm{b}], R_{3} \equiv[\mathrm{C}, \mathrm{c}], \ldots$ etc. To clarify the presentation, hereafter, the conjugate FOSSIL code trees constructed from [A, -a], [B, -b], [B, -c], etc. are denoted by $S F T_{1}, S F T_{2}, \ldots$, etc., respectively, to reflect their relationships to the FOSSIL code trees $F T_{1}, F T_{2}, \ldots$, etc., which have root codes $R_{1}=[\mathrm{A}, \mathrm{a}], R_{2}=$ $[\mathrm{B}, \mathrm{b}], R_{3} \equiv[\mathrm{C}, \mathrm{c}]$, etc., respectively.

We use a special indexing rule to refer to the codes in the conjugate FOSSIL. Let $C_{r}^{i}=[\mathrm{A}, \mathrm{a}]$ be a root code in a FOSSIL. (The length of this sequence is $r$, and $i$ is the index referring to its position in the FOSSIL.) Then, $[\mathrm{A},-\mathrm{a}]$ is a root in the conjugate FOSSIL of the FOSSIL. We denote this conjugate FOSSIL's root corresponding to FOSSIL's root as $S C_{r}^{i}$. For an arbitrary node of FOSSIL, $C_{n}^{j}$, its first-boron and second-born children are indexed as $C_{2 n}{ }^{2 j}$ and $C_{2 n}{ }^{2 j-1}$, respectively. In the case of the conjugate FOSSIL, the first-born and the secondborn of an arbitrary node $S C_{m}{ }^{k}$ are indexed as $S C_{2 m}{ }^{2 k-1}$ and $S C_{2 m}{ }^{2 k}$, respectively. (Note the difference of the conjugate FOSIIL nodes' indexing rule!) These relationships of FOSSIL and its conjugate FOSSIL are illustrated by the example in Fig. 6. In Fig. 6, note that the code index (not the code sequence) in conjugate FOSSIL increases from left to right.

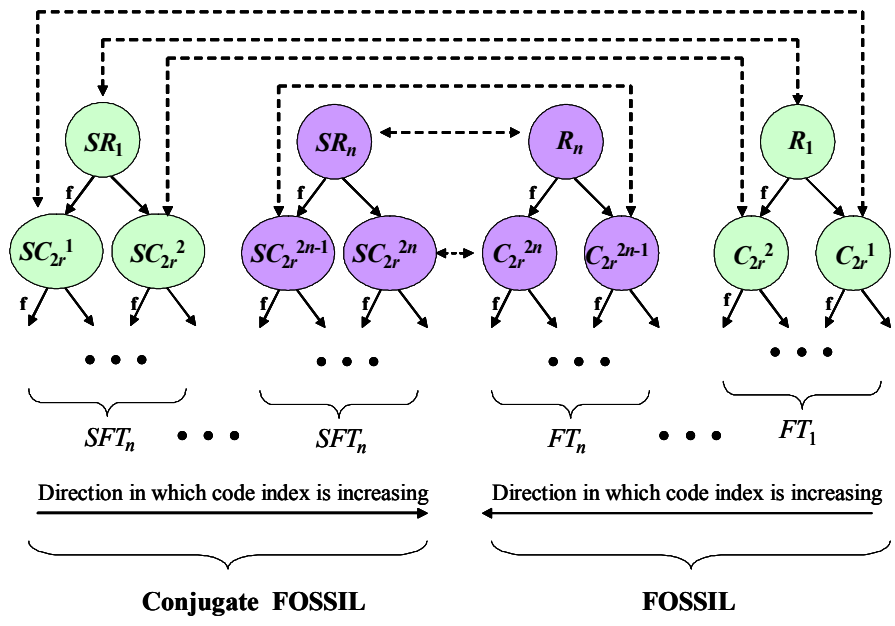

Figure 6. A FOSSIL and its conjugate FOSSIL (" $\mathrm{f}$ " refers to the first-born.)

Under this special arrangement of the conjugate-FOSSIL's code index, it can be shown that the conjugate-FOSSIL code $S C_{n}{ }^{i}$ has sequences $\left[{ }_{1} C_{n}{ }^{i},-{ }_{2} C_{n}{ }^{i}\right]$ where $C_{n}{ }^{i}=\left[{ }_{1} C_{n}{ }^{i},{ }_{2} C_{n}{ }^{i}\right]$. In other words, $S C_{n}^{i}$ and $C_{n}{ }^{i}$ are both constructed by the same two subsequences ${ }_{1} C_{n}{ }^{i}$ and ${ }_{2} C_{n}{ }^{i}$. In this paper we call $S C_{n}{ }^{i}$ a conjugate code (node) of $C_{n}{ }^{i}$. The conjugate relation between a FOSSIL node and its conjugate FOSSIL node is illustrated by the dashed lines between FOSSIL and Conjugate FOSSIL nodes in Fig. 6. Fig. 7 provides an example of a FOSSIL and its conjugate FOSSIL. 

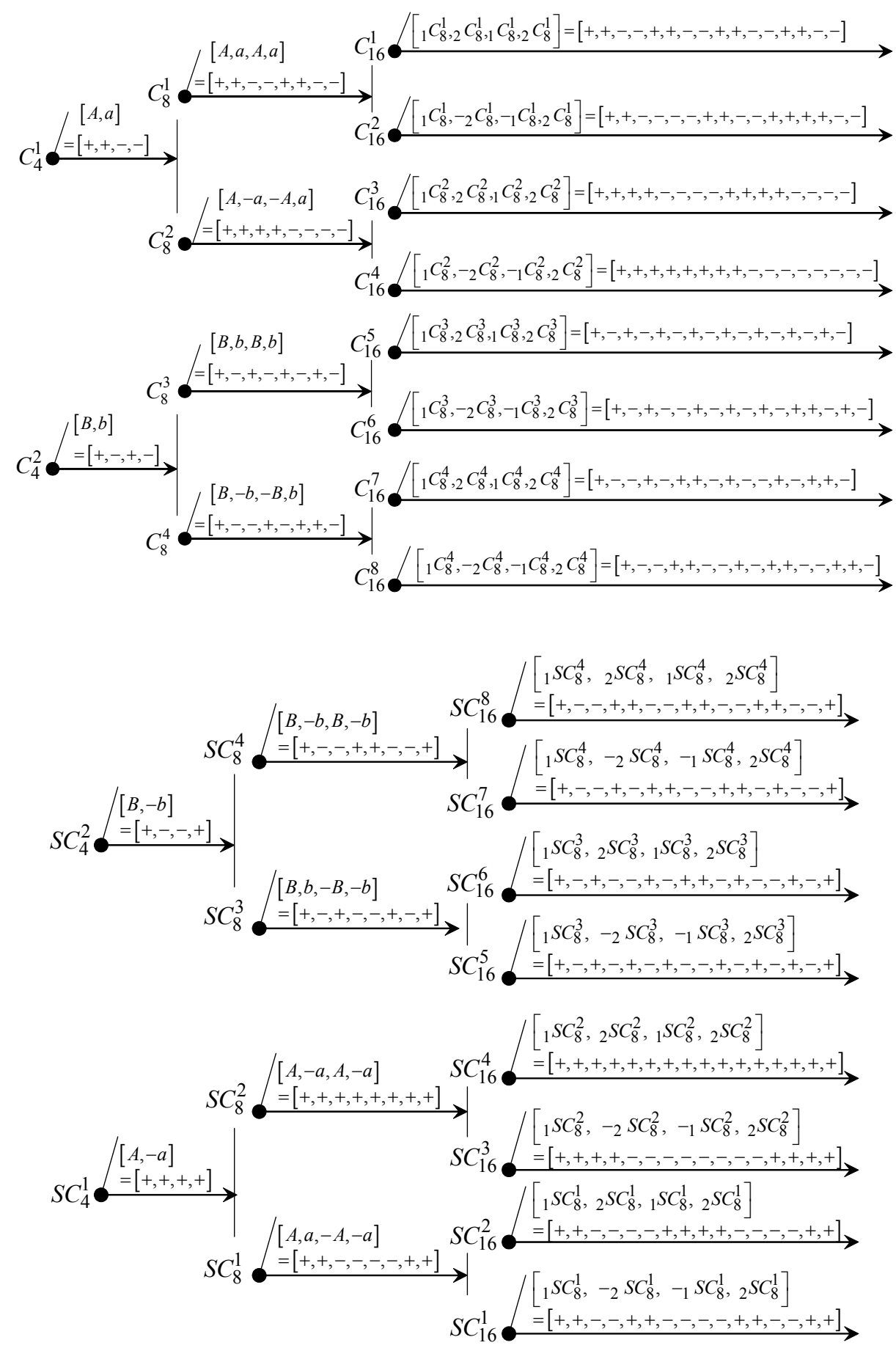

Figure 7 Example of FOSSIL and its conjugate FOSSIL

\section{MULTI-USER MULTI-RATE ADAPTIVE SYSTEM WITH FEEDBACK}

An example use of the symbol-by-symbol SG adaptation would be the transmitter's rapid adaptation to the channel condition. In this application, however, the transmitter needs rapid feedback of the channel condition from the receiver. For symbol-by-symbol adaptation to be effective, the feedback needs to be almost as often as the symbol transmission. In this section, we add such a fast feedback capability to the rapid spreading adaptation system introduced in section II.

The scheme to be introduced in this section operates in a full duplex communication system and allows both station's transmitters to rapidly adjust their SGs in response to the peer stations' feedback information. For example, the feedback information can be the receiver's recommendation to the transmitter to increase/decrease the symbol duration (spreading gain) by $0 \mathrm{~dB}, 3 \mathrm{~dB}, 6 \mathrm{~dB}$, etc. As the $\mathrm{SG}$ of the currently symbol is indicated implicitly by the spreading code, the receiver's feedback information is indicated by its choice of the spreading code used for data symbol carried in the reverse link. A transmitter in each side of the full duplex link uses two sets of spreading codes: one for indicating the spreading gain of the symbol being transmitted and the other for indicating the feedback information. We refer to these codes sets as RI (rate information) code set, $\Psi_{\mathrm{RI}}$, and FBI (feedback information) code set, $\Psi_{\mathrm{FBI}}$. The transmitter spreads each symbol either by a code in RI code set or a code in FBI code set. We refer to a symbol 
spread by an RI code by an "RI-symbol" and a symbol spread by an FBI code by an "FBI-symbol". The role of an RI code set can be viewed as that of the code set $\mathcal{S}$ used in section II (essentially a code set with IOVSF property). The feedback information at a given moment is carried by the choice of an FBI code from the FBI code set to spread the FBI symbol at the moment. A station gives feedback information to the other side by interjecting FBIsymbols between some RI-symbols. A station receiving a signal obtains the feedback information sent from the other station by deciding the FBI code used to spread the FBI-symbol being received.

The receiver detects (again by correlating with possible spreading codes) the current symbol duration, whether the current symbol is an RI-symbol or an FBI-symbol, and what FBI code has been used if the current symbol is an FBI symbol. The detection method is presented in section IV.C. To enable the receiver's detection, The RI code set and the FBI code set used together must collectively satisfy some properties. Section IV.A describes how to construct these code sets. Again to enable the receiver's detection without compromising an extremely rapid spreading gain adaptation, we impose a certain temporal rule to be observed by the transmitter in selecting the spreading gain and in deciding whether to send an RI-symbol or an FBI-symbol. Section IV.B describes the temporal rule.

\section{A. Construction of RI code set and FBI code set}

In order to give a link a range of spreading gain from $p$ to $2^{L-1} p$, we can select a code with length $p$ in FOSIIL and add its $L-1$ descendants along the first-born lineage to construct an RI set; e.g., $\Psi_{R I}=\left\{C_{k p}{ }^{k \alpha} \mid k=1,2,4, \ldots, 2^{L-1}\right\}$. (In a multi-user environment, the RI code sets for different forward links must be chosen in the way discussed in section III.B in order to preserve signal orthogonality among the links.)

An FBI set for the link must be constructed in association with an RI set. For convenience of notation, we denote by $C_{p}^{\alpha}$ the shortest code in $\Psi_{R I}$ and denote

$$
R I_{j} \equiv C_{2^{j} p}^{2^{j} \alpha}, j=0,1,2,3, . ., L-1
$$

for this illustration. In order to discuss this construction, we note in advance that the temporal rule to be presented in section $\mathrm{B}$ allows the transmitter to send an FBI symbol only immediately after sending an RI symbol. Moreover, the choice of FBI code that can be used immediately after an RI symbol $R I_{j}$ is limited. We denote by $\Phi_{F B I}(j) \equiv\left\{F_{j, 0}, F_{j, 1}, \ldots, F_{j, m-1}\right\}$ (where $m$ $=3$ for $j=0$ and $m=2^{j+1}-1$ otherwise) the set of FBI codes that can be used by the transmitter immediately after using $R I_{j}$. Then, $\Phi_{F B I}(j)$ is constructed from the FOSSIL and its conjugate FOSSIL according to:

1. For $j=1,2, \ldots, L-1, m$ is set to $2^{j+1}-1$. Codes in $\Phi_{F B I}(j)$ are (where $k \equiv 2^{j}$ ),

$F_{j, 0}=S C_{k p}{ }^{k(\alpha-1)+1}$

$F_{j, 1}=S C_{k p}^{k(\alpha-1)+2}$

$\cdots$

$F_{j, k / 2-2}=S C_{k p}^{k \alpha-1}$

$F_{j, k / 2-1}=C_{k p}^{k \alpha}$

$F_{j, k / 2}=C_{k p}{ }^{k-1}$

$F_{j, m-1}=C_{k p}{ }^{k(\alpha-1)+1}$

(Note that $S C_{k p}{ }^{k \alpha}$ is not in $\Phi_{F B I}(j)$.)
2. For $j=0, m$ is set to 3 . Codes in $\Phi_{F B I}(0)$ are, $F_{0,0}=S C_{2 p}^{2 \alpha-1}$

$F_{0,1}=C_{2 p}{ }^{2 \alpha}$

$F_{0,2}=C_{2 p}{ }^{2 \alpha-1}$

(Note that $\Phi_{F B I}(0)=\Phi_{F B I}(1)$.)

The FBI code set $\Psi_{F B I}$ associate with $\Psi_{R I}$ is then defined as $\bigcup_{j=0}^{L-1} \Phi_{F B I}(j)$. From this construction, we observe a few aspects of the feedback-enabling protocol. The spreading gain of the FBI symbol must be equal to the spreading gain of the immediately preceding RI symbol, with the exception of RI symbol spread by $R I_{0}$. The number of available FBI codes immediately after using an RI code $R I_{j}$ for $j>0$, whose spreading gain is $2^{j} p$, is $2^{j+1}-1$. The number of available FBI codes immediately after using an RI code $R I_{0}$ is 3 . When spreading a data symbol by a code in $\Phi_{F B I}(j)$, the transmitter presents the $m$-ary (where $m=3$ for $j=0$, and $m=2^{j+1}-1$ otherwise) feedback message to the peer station by the choice of a code from set $\Phi_{F B I}(j)$. The mapping between the FBI codes and the feedback messages is a design dependent issue. Section D provides an example of such a mapping in the course of discussion.

The FBI code set associated with RI code set $\Psi_{R I}=\left\{C_{k p}{ }^{k \alpha} \mid k=\right.$ $\left.1,2,4 \ldots, 2^{L-1}\right\}$ includes all codes in $\Psi_{R I}$. The relationship between $\Psi_{R I}$ and $\Psi_{F B I}$ is better illustrated by Fig. 8, where for example, $C_{2 p}{ }^{2 \alpha}$ is in both $\Psi_{R I}$ and $\Psi_{F B I}$. (Thus, the receiver will have to rely on the temporal rules in section $\mathrm{B}$ to decide whether the detected code $C_{2 p}{ }^{2 \alpha}$ used by the transmitter as an RI or an FBI code.) Moreover, $\Psi_{F B I}$ includes all the codes in the smallest branch containing $\Psi_{R I}$ in the FOSSIL and some of their counterparts in the conjugate FOSSIL.

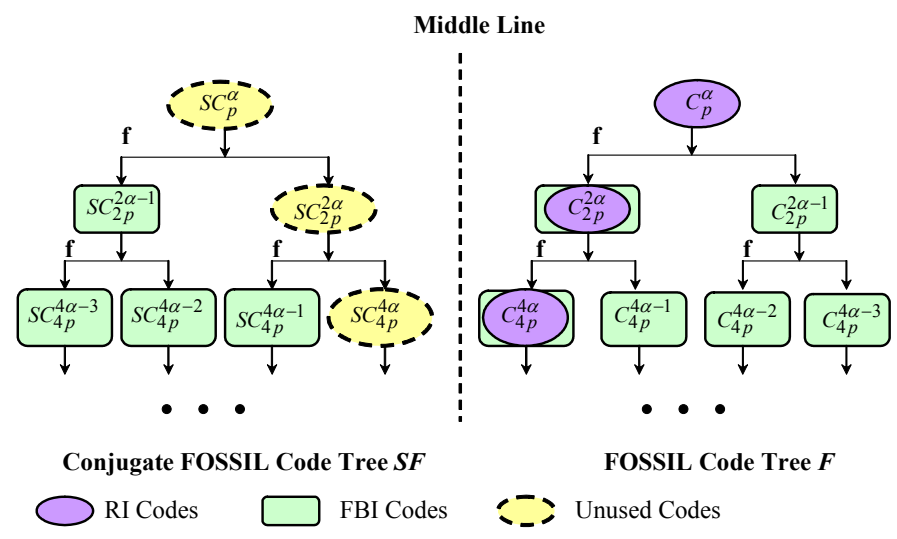

Figure 8. RI and FBI code sets

FOSSIL's Property F3 stated in section III.A and conjugate relations between some pairs of nodes in $\Phi_{F B I}(j)$ proves the following property of $\Phi_{F B I}(j)$.

Property of FBI codes: For each $j$, all codes in $\Phi_{F B I}(j)$ are orthogonal to one another.

\section{B. Protocol at Transmitter Side}

The temporal rules presented in this section have the following design goals: 
1. Obey Rule 1 (modulo counter rule, section II.A) to simplify the receiver and to maintain signal orthogonality among multiple forward links (section II.B).

2. Enable the transmitter to change the SG rapidly.

3. Prevent the receiver from facing un-resolvable ambiguity of what spreading code has been used.

We let RI codes denoted by (5) for our description. As alluded in section $\mathrm{A}$, the transmitter has a rule:

- Immediately after transmitting an FBI symbol, the transmitter must use an RI code to spread the next symbol.

Regarding the RI symbol that immediately follows an FBI symbol, the transmitter has freedom to use any RI code as long as the RI code does not violate the 'modulo counter' rule. (This choice of RI code determines the spreading gain, and this choice can be made intelligently based on the feedback information on the channel and/or the application's rate adaptation policy.)

Immediately after transmitting an RI symbol, the transmitter has freedom and restrictions in its use of spreading codes for the next symbol. We now discuss this rule. We discuss choices and restrictions of the transmitter in choosing a spreading code for a symbol that immediately follows an RI symbol spread by $R I_{j}$. Note that immediately before or after transmitting an RI symbol spread by $R I_{j}$, the modulo counter $Y$ is at $\left(Y \bmod \left|R I_{j}\right|\right)=0$.

1) Case $j>0$

In this case the rule can be summarized as the following:

- If the duration of the next symbol cannot strictly increase (due to the modulo counter rule or because $R I_{j}$ is the longest code in the RI code set), the next symbol must be an FBI symbol spread by a code in $\Phi_{F B I}(j)$.

- If the duration of the next symbol can strictly increase, the next symbol can be either an FBI symbol spread by a code in $\Phi_{F B I}(j)$ or an RI symbol with strictly longer duration.

We now discuss this in more details. Suppose $j=L-1$; that is, the duration of the current symbol is the longest. Then, the transmitter cannot increase the symbol duration. The next symbol must be an FBI symbol whose spreading code is selected from $\Phi_{F B I}(L-1)$.

Now we discuss case $0<j<L-1$. In this case, the local station must encode the next data symbol by an FBI codeword in $\Phi_{F B I}(j)$ if $\left(Y \bmod \left|R I_{j+1}\right|\right) \neq 0$. (That is, if the transmitter cannot increase the duration of the next symbol due to the modulo-counter restriction discussed in section II, that symbol must be spread by a FBI code with length identical to that of $R I_{j}$.) The associated rule is illustrated by Fig. 9. On the other hand, if $\left(Y \bmod \left|R I_{j+1}\right|\right)=0$, the local station can spread the next symbol by any FBI code in $\Phi_{F B I}(j)$ or spread the following symbol by any longer RI-codeword $R I_{j+l}$ (with $l>0, j+l<L$ ) as long as $\left(Y \bmod \left|R I_{j+l}\right|\right)=0$. In other words, if $\left(Y \bmod \left|R I_{j+l}\right|\right)=0$, the local station can even abruptly increase the symbol duration to that corresponding to the spreading gain of $R I_{j+l}$. This situation is illustrated by Fig. 10 .

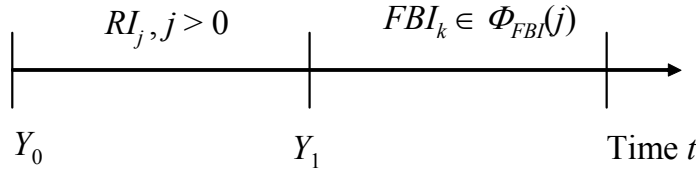

Figure 9. Code transition rules: if $\left(Y_{1} \bmod \left|R I_{j+1}\right|\right) \neq 0$

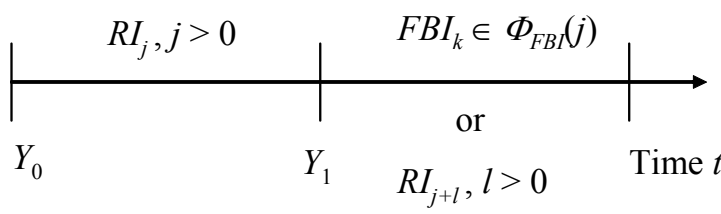

Figure 10. Code transition rules: for $\left(Y_{1} \bmod \left|R I_{j+l}\right|\right)=0$, for $l>0$

\section{2) Case $j=0$}

Suppose that the transmitter has just sent an RI symbol spread by $R I_{0}$ immediately after an FBI symbol. At this state, the rule forces the transmitter to execute the following operations consecutively:

1. Transmit another RI symbol spread by $R I_{0}$.

2. Transmit an FBI symbol spread by a spreading code in $\Phi_{F B I}(0)$.

(Note that $\Phi_{F B I}(0)=\Phi_{F B I}(1)$, and Fig. 11 illustrates this sequential operations.)

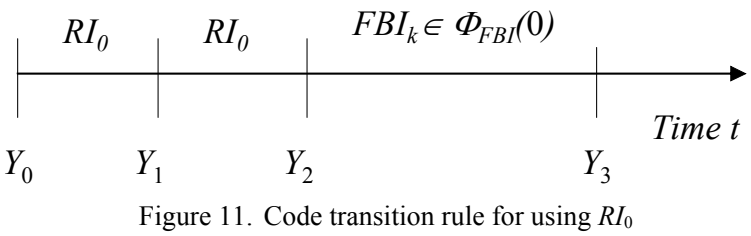

\section{Protocol at Receiver Side}

Due to the temporal rules in section $\mathrm{B}$, the receiver can limit the number of hypotheses on what spreading codes has been used by the transmitter for the current symbol. From among the hypotheses, the receiver decides one by correlating the received signal with the codes corresponding to these hypotheses. Eq. (4) formulates the correlation output. These possible codes are all mutually shift-orthogonal in accordance with the temporal rule of the transmitter and the construction of the RI code set and the FBI code set. Thus, in accordance with section II, the receiver can apply detection schemes to decide the transmitter's choice of the current spreading code. The detection scheme is a design variable and should depend on the system environments. An exemplary detection scheme would be the maximum likelihood detection.

Now we describe the receiver's detection operation in more details.

\section{1) After detecting an FBI symbol}

The next symbol can only be an RI symbol, and it could have been spread by any spreading code in the RI codes set $\Psi_{R I}$ that does not violate the modulo counter rule. The receiver correlates (as in section II.A) the subsequent signal with all RI codes $R I_{j}$ $\in \Psi_{R I}$ such that $\left(Y \bmod \left|R I_{j}\right|\right)=0$. 
Code sequences in $\Psi_{R I}$ are shift-orthogonal to one another, so the receiver can detect the code that has been used by the transmitter.

\section{2) After detecting an RI symbol spread by $R I_{L-1}$}

The next symbol can only be an FBI symbol spread by one of the spreading codes in $\Phi_{F B I}(L-1)$. The receiver correlates the subsequent signal with all spreading codes in $\Phi_{F B I}(L-1)$ to detect which codes has been used (i. e., to extract the feedback information sent from the transmitter regarding the reverse link).

According to Property of FBI codes stated in section A all codes in $\Phi_{F B I}(L-1)$ are orthogonal to one another. Therefore, the receiver can detect the FBI code that has been used by the transmitter.

$$
\begin{aligned}
& \text { 3) After detecting an } R I \text { symbol spread by } R I_{j} \text {, } \\
& 0<j<L-1
\end{aligned}
$$

- If $\left(Y \bmod \left|R I_{j+1}\right|\right) \neq 0$, the next symbol can only be an FBI symbol spread by a code in $\Phi_{F B I}(j)$. Receiver correlates the subsequent signal with all codes in $\Phi_{F B I}(j)$ to extract the feedback information. (all codes in $\Phi_{F B I}(j)$ are orthogonal to one another as stated in section A.)

- If $\left(Y \bmod \left|R I_{j+1}\right|\right)=0$, the next symbol can by either an FBI symbol spread by a code in $\Phi_{F B I}(j)$ or an RI symbol spread by any RI code $R I_{j+l}$ such that $l>0$ and $\left(Y \bmod \left|R I_{j+l}\right|\right)=0$. The receiver correlates the subsequent signal with all codes in $\Phi_{F B I}(j)$ and all RI codes $R I_{j+l}$ such that $l>0$ and $\left(Y \bmod \left|R I_{j+l}\right|\right)=0$. (Codes in $\Phi_{F B I}(j)$ and codes in $\left\{R I_{j+l} \mid l>0, j+l<L\right\}$ are all shift-orthogonal to one another in accordance with Property T1, Property F2, Property F3 stated in section III.A and the conjugate relations of codes stated in III.C.)

\section{4) After detecting an RI symbol spread by $R_{0}$ immediately} following an FBI symbol

Due to the transmitter-side protocol in section $\mathrm{B}$, another RI symbol spread by $R I_{0}$ and an FBI symbol must follow in sequence. The receiver correlates the subsequent signal by $R I_{0}$ and then all the codes in $\Phi_{F B I}(0)$. (The first correlation with $R I_{0}$ is not essential but can be used to examine whether the receiver is maintaining modulo counter synchronization with the transmitter. The receiver can lose synchronization due to previous detection error.)

\section{Comments on Protocol}

Note that the protocol forbids the transmitter from spreading two consecutive RI-symbols with the second symbol duration shorter than or equal to the first ${ }^{3}$ with the exception of the transmitter's rule in section B for $j=0$. (This rule for $j=0$ is an exception designed for sharing of the FBI codes, $\Phi_{F B I}(0)=$ $\Phi_{F B I}(1)$.) However, the protocol allows the transmitter to spread consecutive symbols of increasing length with RI codes of

\footnotetext{
${ }^{3}$ This is not to say that two consecutive symbols cannot be both spread by the same spreading code $R I_{j}$. In fact, according to our code set construction, $R I_{j}$ is both in $\Psi_{R I}$ and $\Phi_{F B I}(j)$. An RI symbol spread by $R I_{j}$ can be followed by an FBI symbol that happens to be spread by the same sequence $R I_{j}$ in the FBI code set. In this case, the receiver interprets the second symbol as an FBI symbol.
}

increasing length as long as modulo counter condition is not violated. ${ }^{4}$ This way, we allow transmitter to rapidly increase symbol durations (possibly to adapt to bad channel condition rapidly). Note that at some protocol states, the transmitter has an option between transmitting a longer RI-symbol and transmitting an FBI-symbol of the equal length. Thus, a station has some control on how often it sends feed back information to its peer station. A station could delay on reporting the reverse link's condition to its peer. A station's decision on how often it sends feedback information affects the data transmission through the link in the reverse direction.

There are several conceivable reasons why a station may prefer transmitting a symbol with longer RI-code instead of an FBI code at some points. To consider these, let us assume that stations agree to interpret the feedback information carried by the choice of an FBI code as "a request to change" the spreading gain by ' $3 \mathrm{~dB}$ ', ' $-3 \mathrm{~dB}$ ', ' $6 \mathrm{~dB}$ ', ' $-6 \mathrm{~dB}$ ', ... Then, if the reverse channel's condition stays roughly the same, no feedback is required. Or, a station may have confidence that the reverse link will stay sufficiently good for a while (possibly based on extremely high strength of the currently received signal) and still do not wish to increase the incoming symbol rate. Then, the station can hold off sending feedback information. There is an interesting tradeoff between sending feedback information and sending an RI symbol with increased spreading gain. After sending a longer RI symbol, the subsequent FBI symbol has a 'larger' alphabet size. Thus, by holding off feedback, a station can send 'more' feedback information in the near future. For example, when an FBI symbol can use codes in $\Phi_{F B I}(1)$, the alphabet size is 3 , so feedback signaling can be defined as carrying messages, "change the spreading gain by $\{-3 \mathrm{~dB}, 3, \mathrm{~dB}$, $6 \mathrm{~dB}$ ?", for example. After sending and RI symbol spread by $R I_{2}$ instead of an FBI symbol, an FBI symbol can be spread by codes in $\Phi_{F B I}(2)$, which has alphabet size 7 . Then, the feedback signaling may be defined as carrying messages "change the spreading gain by $\{-9 \mathrm{~dB},-6 \mathrm{~dB},-3 \mathrm{~dB}, 3 \mathrm{~dB}, 6 \mathrm{~dB}, 9 \mathrm{~dB}, 12$ $\mathrm{dB}\}$ ". The transmitter's proper decision-making strategy between a longer RI-symbol or a FBI-symbol is not within this paper's scope and will be left as a subject for future study.

For the receiver to properly operate, the receiver's modulo counter must be in proper synchronization with the transmitter's. Such synchronization can be achieved by adding a modulo counter synchronization mechanism be added at the link initialization stage. We have designed a few modulo counter synchronization protocols, and Appendix A sketches one.

\section{DISCUSSIONS}

This paper has presented a fast symbol-by-symbol spreading gain control protocol. The protocol does not exchange explicit messages on the spreading gain to be used, yet the receiver can detect the spreading gain of the symbols. The protocol for full duplex links allows extremely fast feedback (e.g., of the channel condition) from the receiver side to the transmitter side. Under fading channel models, the symbol-by-symbol spreading gain adaptation can increase data throughput by many orders of magnitude for a given symbol error rate requirement [23]. Thus, we believe that further study of the symbol-by-symbol adaptation offers promise. We are optimizing the protocol architecture and adding more features to improve the operation's robustness. Our

\footnotetext{
${ }^{4}$ If this modulo counter condition is not satisfied, the local station must transmit an FBI-symbol instead.
} 
study [23] indicates that the throughput gain will be extremely high if the stations employ a high carrier frequency and/or if they move at a high speed. This property comes from the relation between the time-varying nature of fading and the Doppler shift [27][28][29].

Our presentation often used the term 'forward links' to refer

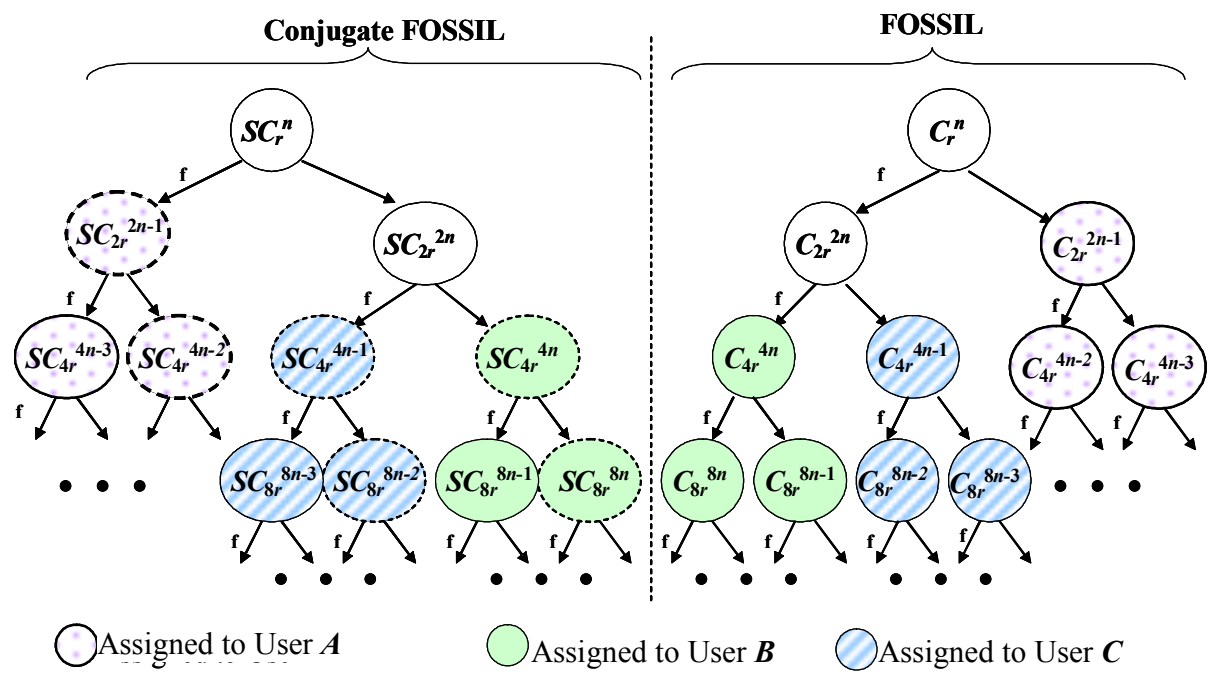

simple temporal rules in section IV to spread their own data symbols, then the data symbols for each user can be extracted out due to the shift-orthogonality of the spreading codes in different partitions. As a result, the information pipe is divided into multiple isolated data links for different users.

We note here that our scheme can be used for uplink transmissions as well. In the case of uplinks, the spreading codes cannot be used to maintain orthogonality of different user signals because we cannot synchronize the mobile station's symbol transmission times. However, if the user signals are separated by pseudo random codes, the OVSF spreading codes in the FOSSIL/conjugate FOSSIL along with our rapid spreading gain adaptation scheme with feedback can still be used for efficient uplink transmissions through rapid rate adaptation. In fact, our architecture can be used for any point-to-point link whose signal is distinguished from the signals of other users in the frequency-time-code signal space, although the OVSF codes' channelization capability will not be utilized in such a case.

Fig. 12. Partition of FOSSIL and conjugate FOSSIL for multiple users

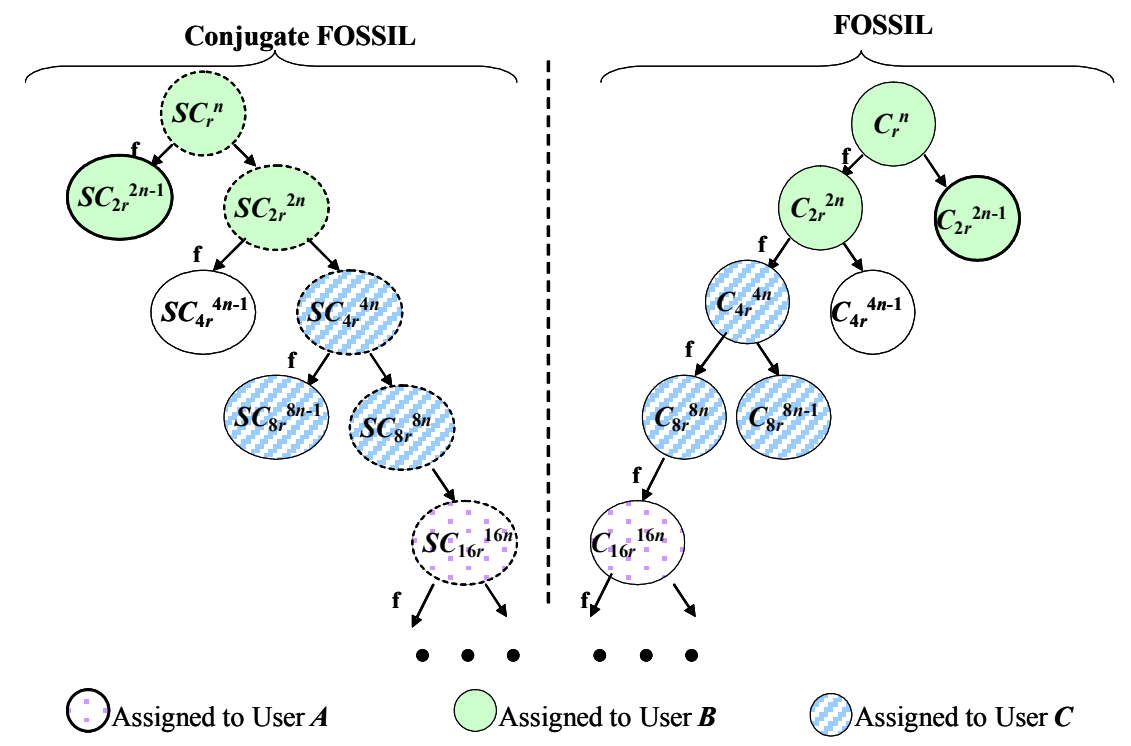

Fig. 13. Partition for users with different ranges of data rates

to multiple links in point-to-multipoint systems. As the term indicates, our rapid spreading gain adaptation and feedback schemes can readily be used for downlink transmissions of cellular networks. If the OVSF codes are used for channelizing different downlinks (separation of different users' signals), our schemes can be applied by properly partitioning the codes in a FOSSIL and its conjugate FOSSIL so that the codes in different partitions are shift orthogonal. Fig. 12 and Fig. 13 provide examples of such partitions for different links sharing the same radio spectrum. FOSSIL properties T1, F2, F3, F4 mentioned in section III make the codes assigned to different users shiftorthogonal. If all the code spreaders at the base station, where a universal modulo counter $Y$ in section II is maintained, follow the

\section{APPENDIX A: INITIALIZATION PROCEDURE OF THE MODULO-COUNTERS}

In this appendix we provide a simple procedure by which stations can initialize the modulocounters over the full duplex link $^{5}$ in a general direct-sequence spread spectrum (DS-SS) communication infrastructure, where any station can initiate the communication link to the other. We assume OVSF/PN system, in which the transmitted signal's waveform is first spread by the OVSF codes (RI codes and FBI codes in this paper) and subsequently modulated by a PN code (which we also refer to as scrambling code) during the normal operation ${ }^{6}$. We further assume that the OVSF chip duration and the PN chip duration are aligned with each other. The spreading process of the system is illustrated in Fig. 3. During the link initialization process (prior to normal operation), the data sequence is spread only by the PN sequence and not by the OVSF sequences. Here, without providing detailed description, we simply refer this signal to as the 'preamble'. (The content of the preamble is not important for synchronizing our modulo counter. For example, the preamble sequence can be symbols of constant value. The transmitter has to transmit some signal with positive power for a period of time in order to assist the receiver to obtain necessary information for synchronization.)

\footnotetext{
${ }^{5}$ For Station $A$ and $B$ operating in the full-duplex link, two pairs of modulocounters are used; (transmitter of $A$ - receiver of $B$ ) pair and (transmitter of $B-$ receiver of $A$ ) pair. Each pair must be synchronized with each other.

${ }^{6}$ The normal operations are different from those performed during the initialization phase.
} 
When a station sends its preamble spread by the PN sequence, the receiver will be able to acquire the signal timing of the PN sequence. The method of such acquisition is beyond the scope of the present paper, but the reference [25] provides good descriptions. We now present a modulo counter synchronizing protocol to be added to this acquisition process. This protocol uses a special code (chip sequence) $C N T-S I G$ to synchronize the modulo counter. The length of this code is an integer multiple of the length of the longest RI code. Basically, each station's transmitter spreads data with this special code (in place of the OVSF codes in FOSSIL or conjugate FOSSIL) and sets its modulo counter to 0 at the end of this code, and starts the normal transmission operation using OVSF codes. The receiver of the other station acquires the end time of this CNT-SIG through correlation and sets its modulo counter to 0 at the end of this code. Thus, the receiver synchronizes its modulo counter with the transmitter of the other station. We now describe the procedure in more details.

The station that is initiating communication first transmits preamble and monitors the peer station's preamble in order to acquire the PN sequence's signal timing of the incoming signals. We refer to this state as 'begin' state; i.e., the state of transmitting the preamble and trying to acquire the PN code chip timing. At either station, after acquisition of the incoming signal's PN sequence timing (i.e., after entering 'acquired' state) does the following:

\section{Initialization Procedure A.1}

After acquisition of the PN signal timing from the other party, the station does the following:

1) Transmitter - Its transmitter transmits the preamble for a fixed time duration $\Delta_{l}$; then, transmit a special code CNT-SIG; at the end of CNT-SIG, the transmitter's modulo counter is set to 0 , and the normal transmission operation begins.

2) Receiver - Its receiver starts correlating the received signal with CNT-SIG sequence after descrambling the PN code. When acquiring the end of CNT-SIG sequence, the receiver's modulo counter is set to 0 and the normal receiving operation begins.

The timing diagram of the above simple initialization procedure is illustrated in Fig. 14 and Fig. 15.

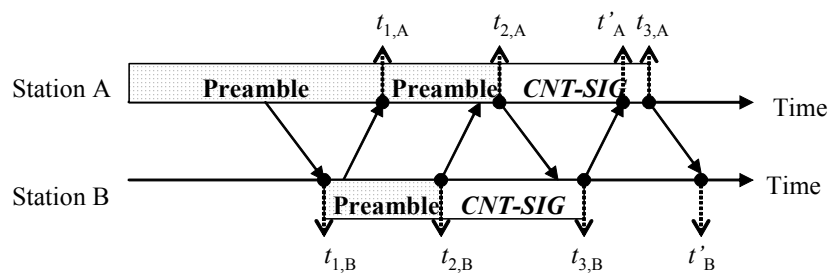

Figure 14. Station A starting the initialization process

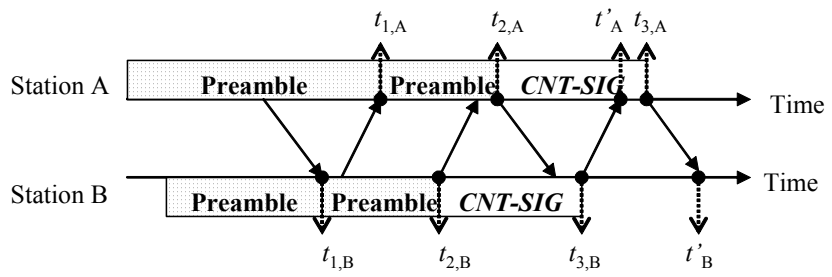

Figure 15. Stations A and B concurrently starting the initialization process
After acquisition, a station transmits the preamble for another $\Delta_{1}$ seconds. The reason is to give the other station sufficient chance to acquire the PN sequence timing. For example, in Fig. 14 , station $B$ does not initiate communication. After station $B$ acquires the PN sequence timing of the signal incoming from station $A$, station $B$ must send its preamble spread by its PN sequence to station $A$ so that station $A$ can acquire the PN sequence timing of the signal coming from station $B$. It can be easily seen in Fig. 15 that the procedure works fine even if both stations happen to initiate communication concurrently.

$\Delta_{1}$ time units after acquisition, the station's transmitter starts spreading the data (which can be null data) with a special sequence, CNT-SIG. The length of CNT-SIG, $\Delta_{2}$ is an integer multiple of the longest RI code. At the end of the last CNT-SIG chip, the transmitter sets its modulo counter $Y$ to 0 .

At each station, after acquiring the PN sequence timing (at $t_{1, \mathrm{~A}}$ for Station $A$ and $t_{1, \mathrm{~B}}$ for Station $B$ in Fig. 14.), the receiver starts searching for the signature's waveform of the CNT-SIG code, expecting that a signal encoded by this code will soon be coming in. For every correlation, the signature pulse train generated by the receiver will be linen up with the received signal. Thus, if the receiver acquires the ending point of the CNT-SIG sequence, the modulo counter can be synchronized by mandating the transmitter and the peer receiver to reset their own modulo counters to zero at the end of CNT-SIG sequence.

In this discussion, we do not intend to specify the design of CNT-SIG sequence in detail. A requirement is that the receiver can detect this sequence in the incoming chip stream. However, we introduce a system design that makes the design of CNT-SIG sequence much simpler while making the synchronization mechanism more robust. That is, we mandate that the transmitter starts the CNT-SIG sequence only at a specified position of the periodic [25][26] PN sequence. Because the receiver attempts to detect CNT-SIG sequence only after acquiring the PN sequence timing, this additional rule enables to the receiver to preeliminate many chip positions at which the CNT-SIG sequence can begin, so the detection of $C N T-S I G$ sequence becomes very simple.

In the ideal cases shown in Fig. 14, station A's and B's receivers can properly reset their own modulo counters after successfully detecting $C N T-S I G$ from the other stations. When operating over a noisy (unreliable) channel, the receiver in each link may miss the $C N T-S I G$. To prevent the stations from trying to detect $C N T-S I G$ endlessly after missing one, in our design, after acquiring the PN code's chip timing, each station resets its link initialization process if it does not detect $C N T-S I G$ for a given length, $T_{\text {out, }}$ of time. The station should also reset its transmitter to force the other station to fall back to the 'begin' state of detecting preamble. (This can be done by halting the transmission of signal for a given amount of time, $T_{\text {shut, }}$ and start transmitting the preamble spread by the PN code. The other station will judge that the link is broken after observing the absence of incoming signal for $T_{\text {shut }}$ and will try to initialize the link.) The time-out period, $T_{\text {out }}$, must be long enough to include signal's propagation delay $\left(\Delta_{\mathrm{d}}\right)$ and CNT-SIG's duration $\left(\Delta_{2}\right)$, and it is sufficient to set $T_{\text {out }}$ equal to $\left(2 \Delta_{\mathrm{d}}+2 \Delta_{1}+\Delta_{2}\right)$.

\section{REFERENCES}

[1] L. B. Milstein, "Wideband code division multiple access" IEEE $J S A C$, vol. 18, No. 8, pp. 1344-1354, Aug. 2000. 
[2] F. Adachi, M. Sawahashi, and H. Suda, "Wideband DS-CDMA for next-generation mobile communications systems," IEEE Communication Magazine, September 1998, pp. 56-69.

[3] U. Mitra, "Comparison of maximum-likeihood-based detection for two multirate access schemes for CDMA signals," IEEE Transactions on Communications, vol. 47, no. 1, Jan. 1999, pp. 64-77.

[4] E. Dahlman, B. Gudmundson, M. Nilsson, and J. Sköld, "UMTS/IMT-2000 based on wideband CDMA," IEEE Comm. Magazine, pp. 70-80, Sept. 1998.

[5] M. H. Fong, V. K. Bharagva, Q. Wang, "Concatenated orthogonal/PN spreading sequences and their application to cellular DS-CDMA systems with integrated traffic," IEEE $J$. Select. Areas Commun., vol. 14, no3, pp. 547-558, April 1996.

[6] S. W. Golomb, "Construction of signals with favorable correlation properties," London Mathematical Society Lecture Note Series 166, Surveys in Combinatorics 1991, Edited by A. D. Keedwell, pp. $1-39$

[7] P. Jung, P. W. Baier, A. Steil, "Advantages of CDMA and spread spectrum techniques over FDMA and TDMA in cellular mobile radio applications," IEEE Trans. On Vehicular Tech., vol. 42, no. 3, pp. 357-364, Aug. 1993.

[8] J. F. Hayes, "Adaptive feedback communications," IEEE Trans. Commun. Technol., vol. COM-16, pp. 29-34, Feb 1968.

[9] J. K. Cavers, "Variable-rate transmission for Rayleigh fading channels", IEEE Trans. Commun., vol. COM-20, pp. 15-22, Feb. 1972.

[10] B. Vucetic, "An adaptive coding scheme for time-varying channels," IEEE Trans. Commun., vol. 39, pp. 653-663, May 1991.

[11] M.-S. Alouini and A. Goldsmith, "Adaptive M-QAM modulation over Nakagami fading channels," in Proc. Communi. Theory Mini-Conf. (CTMC-VI) in conjuction with IEEE GLOBECOM'97, pp. 218-223, Nov. 1997.

[12] V. O. Hentinen, "Error performance for adaptive transmission on fading channels," IEEE Trans. Commun., vol. COM-22, pp. 13311337, Sept. 1974.

[13] A. Goldsmith, and S. G. Chua, "Adaptive coded modulation for fading channels," IEEE Trans. Commun., vol. 46, pp. 595-602, May 1998.

[14] M.-S. Alouini and X. Tang, A. J. Goldsmith, "An adaptive modulation scheme for simultaneous voice and data transmission over fading channels," IEEE JSAC, vol. 17, no. 5, pp. 837-850, 1999.
[15] S. J. Oh, K. M. Wasserman, "Dynamic spreading gain control in multiservice CDMA networks," IEEE JSAC, vol. 17, no. 5, pp. 918-927, May 1999.

[16] C.-L. I. and K. K. Sabnani, "Variable spreading gain CDMA with adaptive control for true packet switching wireless network," in Proc. of IEEE ICC'95, pp. 725-730, 1995.

[17] T.-H. Lee, and J. T. Wang, "Admission control for variable spreading gain CDMA wireless packet networks," IEEE Trans. On Vehicular Tech., vol. 49, No. 2, pp. 565-575, March 2000.

[18] T. Minn and K. Y. Siu, "Dynamic assignment of orthogonal variable-spreading-factor codes in W-CDMA," IEEE JSAC, vol. 18, No. 8, pp. 1429-1440, Aug. 2000.

[19] Y. Okumura, and F. Adachi, "Variable-rate data transmission with blind rate detection for coherent DS-CDMA mobile radio," IEICE Trans. Comm., vol. E81-B, no. 7, July 1998, pp. 1365-1372.

[20] L. Tsuar and D. C. Lee, "Use of OVSF code for zero-overhead symbol rate adaptation," Proc. of ISMIP'99, December, 1999, pp. 232-241.

[21] F. Adachi, M. Sawahashi, and K. Okawa, "Tree-structured generation of orthogonal spreading codes with different length for forward link of DS-CDMA mobile radio," Elect. Lett., vol. 33, no. 1, Jan. 1997 , pp. 27-28

[22] L. Tsaur and D. Lee, "Symbol rate adaptation and blind rate detection using FOSSIL (Forest for OVSF-Sequence-SetIncluding Lineages)," in Proc. IEEE ICC'01, June 2001, pp. $1754-1759$.

[23] L. Tsaur and D. C. Lee, "Effects of symbol-by-symbol rate adaptation on error-constrained data throughput over multi-path fading channels", CSI Publications CSI-03-12-01 http://wwwrcf.usc.edu/ dclee/publications-year.html.

[24] A. J. Goldsmith, P. P. Varaiya, "Capacity of fading channels with channel side information," IEEE Trans. on Info. Theory, vol. 43, no. 6, pp. 1986-1992, Nov. 1997.

[25] A. J. Viterbi, CDMA Principles of Spread Spectrum Communication, Reading, MA: Addision Wesly, 1994.

[26] S. W. Golomb, Shift Register Sequences. San Francisco: HoldenDay, 1967.

[27] T. S. Rappaport, T. Rappaport, Wireless Communications: Principles and Practice, $2^{\text {nd }}$ ed., Upper Saddle River, NJ: Prentice Hall, 2001.

[28] W. C. Jakes, Microwave Mobile Communications, New York: Wiley, 1974.

[29] G. L. Stüber, Principles of Mobile Communications, $2^{\text {nd }}$ Ed. Boston: Kluwer Academic Press, 2001. 